World Journal of Advanced Research and Reviews
eISSN: 2581-9615 CODEN (USA): WJARAI
Cross Ref DOI: $10.30574 /$ wjarr
JuRR
Journal homepage: https://wjarr.com/

(CASE REPORT)

Check for updates

\title{
Subacute idiopathic pericarditis with a large pericardial effusion in a young adult seven months post COVID-19 (SARS-Cov-2) infection
}

\author{
Ashan T Hatharasinghe 1, ${ }^{*}$ and Andrey E Manov ${ }^{2}$ \\ ${ }^{1}$ DO PGY-3 Internal Medicine Resident Sunrise Health GME Consortium, Mountain View Hospital2880 N Tenaya Way, $2^{\text {nd }}$ \\ Floor Las Vegas, $\mathrm{NV}, 89128$. \\ ${ }^{2}$ Associate Program Director in Internal Medicine Residency Program Sunrise Health GME Consortium, Mountain View \\ Hospital 2880 N Tenaya Way, 2nd Floor Las Vegas, NV, 89128
}

World Journal of Advanced Research and Reviews, 2021, 10(01), 340-342

Publication history: Received on 21 March 2021; revised on 26 April 2021; accepted on 28 April 2021

Article DOI: https://doi.org/10.30574/wjarr.2021.10.1.0187

\begin{abstract}
The number of COVID-19 (SARS-Cov-2) cases has risen substantially throughout the world, and consequently we are finding there are several extrapulmonary manifestations associated with this disease. Viral pericarditis and pericardial effusion have been reported several times in COVID-19 patients, however the majority of these cases occurred during active infection or within a relatively short time frame afterwards. The following case is a young female with only a past medical history of COVID-19 pneumonia, seven months prior, presenting with abdominal pain and progressively worsening dyspnea. She was subsequently found to have a large pericardial effusion without tamponade, but requiring a pericardial window. Gross pathology showed fibrinous pericarditis. It is suspected her symptoms and pericardial effusion resulted from a subacute idiopathic pericarditis likely as post-viral complication of COVID-19.
\end{abstract}

Keywords: COVID-19; Subacute pericarditis; Pericardial effusion; Pericardial window

\section{Introduction}

The COVID-19 (SARS-Cov-2) pandemic has made a surge in the United States throughout the past year. Although our understanding of it and its treatment course have certainly improved, we still have yet to determine what the long-term manifestations will be as COVID-19 is a relatively novel disease. Chronic pulmonary disease is certainly an expected outcome, however there may also be cardiac, hematologic, and other extrapulmonary complications associated with COVID-19. Pericarditis has a low incidence overall, especially in the young female population [1]. But, several cases of viral pericarditis and pericardial effusion have been reported in COVID-19 patients. The majority of these cases are typically during or within six months of a symptomatic COVID-19 infection [2-4]. The following case discusses a young female patient presenting with a large pericardial effusion who was subsequently found to have fibrinous pericarditis. The patient only had a past medical history of COVID-19 pneumonia which occurred more than six months prior to presentation.

\footnotetext{
${ }^{*}$ Corresponding author: Ashan Hatharasinghe

PGY-3 Internal Medicine Resident Sunrise Health GME Consortium, Mountain View Hospital2880 N Tenaya Way, 2nd Floor Las Vegas, NV, 89128.
}

Copyright (C) 2021 Author(s) retain the copyright of this article. This article is published under the terms of the Creative Commons Attribution Liscense 4.0. 


\section{Case report}

A 31 year-old Hispanic female with a history of COVID-19 pneumonia seven months prior and no other underlying medical conditions presented with acute onset right upper quadrant discomfort and epigastric pain. The patient also reported dyspnea on exertion and orthopnea for the past two months. The patient was tachycardic but all other vital signs were unremarkable. A COVID-19 reverse transcriptase polymerase chain reaction (RT-PCR) test on admission was negative. An electrocardiogram (ECG) demonstrated sinus tachycardia with a low voltage QRS complex, but no ischemic changes were present. Troponins were also negative. A computed tomography (CT) scan of the abdomen with contrast was obtained to evaluate the abdominal pain but incidentally showed a large pericardial effusion. The CT scan could not exclude acute cholecystitis however a right upper quadrant abdominal ultrasound was negative. CT angiogram of the chest was then obtained and confirmed a large pericardial effusion measuring up to 3.5 centimeters in thickness and small bilateral pleural effusions. A stat echocardiogram showed no evidence of tamponade physiology with a normal ejection fraction (EF) of 55-60\%. As noted above, the patient had a positive SARS-Cov-2 antigen test seven months prior to this admission. She remained at home during her acute illness and reported symptoms of cough, shortness of breath, and fever lasting for a duration of ten to fourteen days, with complete resolution afterwards.

The patient was then hospitalized approximately five months after initial COVID-19 diagnosis for chest pain and dyspnea. A nuclear stress test at that time was notable for an anterior reversible perfusion defect, however serial troponins were negative. An echocardiogram showed an EF of 55-65\% with no regional wall motion abnormalities. Although no pericardial effusion was reported on the echocardiogram, a CTA of the chest during that admission was notable for a small pericardial effusion. Left heart catheterization was deferred due to patient preference and resolution of chest pain, she was subsequently discharged with outpatient cardiology follow up.

The large pericardial effusion was discovered on this admission approximately seven months after COVID-19 diagnosis and two months after the pericardial effusion was first seen. The patient was evaluated by the cardiothoracic surgery team and underwent a pericardial window with removal of 800-900 milliliters of hemorrhagic effusion, and placement of a pericardial drain. The pericardial fluid cytology was only notable for red blood cells. Cultures yielded no growth and gram staining, including for fungal organisms and acid fast bacilli (AFB) was negative. A sample of the pericardium was sent for gross pathology and was described as dense fibrous tissue with chronic inflammation, fibrin deposition, and hemosiderin-laden macrophages; all consistent with findings typically seen in fibrinous pericarditis. The pericardial drain remained in place for 3 days postoperatively and drained approximately 200 milliliters of fluid. The patient was then discharged with resolution of symptoms after a four day hospital course.

\section{Discussion}

Idiopathic pericarditis is the most common cause of pericarditis and pericardial effusion, and it is typically a post-viral sequela $[5,6]$. The case is a unique presentation of a suspected subacute viral pericarditis resulting from a prior COVID19 infection as the patient was otherwise healthy with no chronic medical conditions. The pericarditis was categorized as subacute based on the chronicity of the symptoms, and the absence of tamponade coupled with the size of the effusion [7]. An effusion this large without tamponade physiology is an indication it had been forming slowly over time. The chest pain and dyspnea started nearly five months after COVID-19 diagnosis, and a small pericardial effusion was initially seen at this time indicating the patient likely had undiagnosed pericarditis for at least two to three months, which gradually worsened resulting in the formation of a larger effusion. Pericardial effusion is a rare finding in COVID19 positive patients, however several cases have reported it particularly during acute illness with associated tamponade $[4,9]$. The pericardial fluid can be serosanguineous or hemorrhagic in viral pericarditis, and current cases of suspected COVID-related effusion have shown to be characteristic of either type [4, 9-10]. Although, a case published in December found the fluid to be exudative [11]. One previous case has been documented showing a similar finding of a hemorrhagic effusion however, in this particular patient with COVID-19, the pericarditis occurred one to two months after a positive test [10]. The gross pathology in our case was notable for hemosiderin-laden macrophages consistent with inflammation due to fibrinous pericarditis. This is the most common gross pathology finding in pericarditis and this was also demonstrated in a prior case report of pericarditis suspected secondary to COVID-19 [10, 12].

It is important to note that it cannot be definitively concluded if the pericarditis was due to COVID-19. The pericardial fluid was not sent for RT-PCR, and ultimately there would need to be histopathological evidence of a viral growth. However, this is not unique to our case as a definitive diagnosis can't be obtained in most instances where viral pericarditis is suspected, henceforth why the term "idiopathic" is appropriate. The patient had no chronic medical conditions and rheumatoid arthritis was not considered due to the absence of joint pain and a normal musculoskeletal exam. Pericarditis as the initial presentation of rheumatoid arthritis is rare, and it typically occurs in patients with 
severe disease progression and arthritis [13]. COVID-19 infection was the only known inciting factor within a reasonable timeframe.

\section{Conclusion}

The long-term manifestations of COVID-19 remain to be determined, but in only a year's time we are beginning to see some of the extrapulmonary complications. The fact that these are occurring in young and healthy populations is concerning, and although more data is certainly needed, it appears that we will likely be dealing with the effects of the COVID-19 pandemic for years to come.

\section{Compliance with ethical standards}

\section{Acknowledgments}

The authors declare no acknowledgments.

\section{Disclosure of conflict of interest}

The authors do not have any conflicts of interest or financial interests to disclose.

\section{Statement of informed consent}

Informed consent was obtained from the individual discussed in the case study.

\section{References}

[1] Kytö V, Sipilä J, Rautava P. Clinical profile and influences on outcomes in patients hospitalized for acute pericarditis. Circulation. 2014; 130(18): 1601-1606.

[2] Sauer F, Dagrenat C, Couppie P, Jochum G, Leddet P. Pericardial effusion in patients with COVID-19: case series. Eur Heart J Case Rep. 2020; 4(FI1): 1-7.

[3] Fox K, Prokup JA, Butson K, Jordan K. Acute Effusive Pericarditis: A Late Complication of COVID-19. Cureus. 2020; 12(7): e9074.

[4] Ejikeme C, Gonzalez M, Elkattawy S, Alyacoub R, Sherer C. Subacute COVID-19 Infection Presenting as Indolent Large Pericardial Effusion. Cureus. 2020; 12(10):e10769.

[5] Vakamudi S, Ho N, Cremer PC. Pericardial Effusions: Causes, Diagnosis, and Management. Prog Cardiovasc Dis. 2017; 59(4): 380-388.

[6] Goldman L, Schafer AI, Hoit BD, Oh JK. Pericardial Diseases. In: Goldman-Cecil Medicine. Philadelphia, PA: Elsevier. 2021; 428-436.

[7] Hoit BD. Pericarditis - Cardiovascular Disorders. Merck Manuals Professional Edition.

[8] Salehi S, Abedi A, Balakrishnan S, Gholamrezanezhad A. Coronavirus Disease 2019 (COVID-19): A Systematic Review of Imaging Findings in 919 Patients. AJR Am J Roentgenol. 2020; 215(1): 87-93.

[9] Cross SS, Suvarna SK. Cardiovascular System. In: Underwood's Pathology: a Clinical Approach. 7th ed. Churchill Livingstone Elsevier; 2019; 242-283.

[10] Amoozgar B, Kaushal V, Mubashar U, Sen S, Yousaf S, Yotsuya M. Symptomatic pericardial effusion in the setting of asymptomatic COVID-19 infection: A case report. Medicine (Baltimore). 2020; 99(37): e22093.

[11] Allam HH, Kinsara AJ, Tuaima T, Alfakih S. Pericardial Fluid in a COVID-19 Patient: Is It Exudate or Transudate?.Eur J Case Rep Intern Med. 2020; 7(6): 001703.

[12] Reddy VB, David O, Spitz D, Haber M, Tan CD, Rodriguez ER. Heart, Pericardium, and Blood Vessels. In: Gattuso's Differential Diagnosis in Surgical Pathology. 4th ed. Philadelphia, PA: Elsevier. 2021; 975-1011.

[13] Voskuyl AE. The heart and cardiovascular manifestations in rheumatoid arthritis. Rheumatology (Oxford). 2006; 45(4): iv4-iv7. 